\title{
Le cœur, une glande endocrine
}

Le cour a son hormone : en effet les cellules des oreillettes cardiaques chez les mammifères sécrètent un peptide de composition connue. Ce facteur natriurétique des oreillettes (FNO) est diurétique et vasodilatateur.

\section{Marc Cantin Jacques Genest}

Institut de recherches cliniques de Montréal

Ce travail a été subventionné par le Conseil de recherches médicales du Canada (subventions de groupe) ainsi que par le Conseil national de recherches du Canada et le Ministère de la science et de la technologie du Québec.

Cet article est une synthèse des contributions des membres du Groupe multidisciplinaire de recherches en hypertension" et de certains de leurs collegues"* à ce nouveau champ de recherche :

-G. Thibault, R. Garcia, 7. Gutkomska, P. Hamet, A. De Léan, E. Schiffrin, O. Kuchel, M.B. Anand-Srivastava, 7. Tremblay, S. Pang, M. Cantin, 7. Genest;

- Laboratoire de neuroendocrinologie moléculaire (Dr M. Chrétien, N.G. Seidah et C. Lazure). Laboratoire de biologie moléculaire (Dr 7. Drouin et $M$. Nemer). Laboratoire de la biologie des peptides ( $\operatorname{Dr} P$. Schiller). Laboratoire des lipoprotéines (anticorps monoclonaux) ( $D r R$. Milne).

\section{ADRESSE, TIRES A PART}

M. Cantin : Institut de recherches cliniques de Montréal, IIo, av. des Pins ouest, Montréal, Québec $\mathrm{H}_{2} \mathrm{~W}_{1} \mathrm{R}_{7}$, Canada.

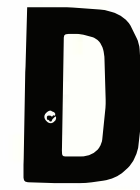

eux voies d'approche différentes ont permis de démontrer que les oreillettes constituent une véritable glande endocrine sécrétant une hormone peptidique. La première méthode s'appuyait sur des concepts d'ordre morphologiques. L'autre forme de recherche a fait appel aux techniques d'investigation physiologique et physiopathologique [1].

Tout d'abord, Kisch a constaté en 1956, la présence de "corps denses " dans les cardiocytes auriculaires du cobaye [2]. Adjacents à un pôle (et occasionnellement aux deux pôles) du noyau, ces "corps denses" sont localisés parmi les citernes, vésicules et saccules d'un complexe de Golgi volumineux. On peut en trouver jusqu'à 600 par cellule chez le rat. Le nombre de granules décroît avec l'augmentation de la taille des animaux. Jamieson et Palade furent les premiers à nommer ces corps denses "granules spécifiques", différents des lysosomes [3]. En 1971, Bencosme et Berger rapportèrent que ces grains étaient aussi présents dans les cardiocytes ventriculaires de nombreux vertébrés non-mammifêres tels que les amphibiens, les reptiles et les oiseaux [4]. Nous avons montré en 1974 que les granules spécifiques étaient riches en protéines [5] et qu'ils incorporaient la leucine et le fucose tritiés comme les autres grains de secrétion des cellules endocrines secrétant des peptides $[6,7]$. Nous avons montré que ces granules ne contiennent ni catécholamine [8] ni rénine [9].

En 1976, Marie et al. étudièrent la granularité des cardiocytes auriculaires chez des rats dont l'apport hydrosodé variait considérablement [10]. Ils trouvèrent que la restric- tion hydrique pendant 5 jours augmente le nombre des granules dans l'oreillette droite sans changement significatif dans l'oreillette gauche. Un régime hypersodé, ainsi que l'administration de déoxycorticostérone et de sodium diminuait de façon significative le nombre de grains dans l'oreillette droite sans changement appréciable dans l'oreillette gauche. La restriction sodée totale pendant 3 semaines augmentait considérablement la granularité dans l'oreillette gauche sans changement dans la droite. Ces résultats ont été confirmés par d'autres chercheurs [11, 12]. En 1980, De Bold, Sonnenberg, Veress et Borenstein [13] démontraient que l'injection d'homogénats d'oreillettes de rats à des rats témoins produisait une diurèse et une natriurèse rapides, massives et de courte durée. D'autres propriétés ont été mises en évidence à la suite de travaux de recherche de caractère physiologique et physiopathologique. Ainsi $a-t$-on pu tenter de préciser les effets de l'augmentation de la pression veineuse intrathoracique, le rôle des réflexes d'origine cardiaque dans l'excrétion d'eau et de sodium et les interrelations entre le volume de liquide extracellulaire, l'équilibre sodé, le volume sanguin et la fonction rénale. Les résultats obtenus ont fourni un schéma général permettant d'interpréter, presque immédiatement, ce qu'il a fallu reconnaître comme une nouvelle fonction cardiaque.

Nous avons démontré que l'activité diurétique et natriurétique des extraits d'oreillettes était localisée dans les granules eux-mêmes [14]. Les essais initiaux de purification ont établi la nature polypeptidique du facteur natriurétique des oreillettes (FNO) et la composition en aci- 


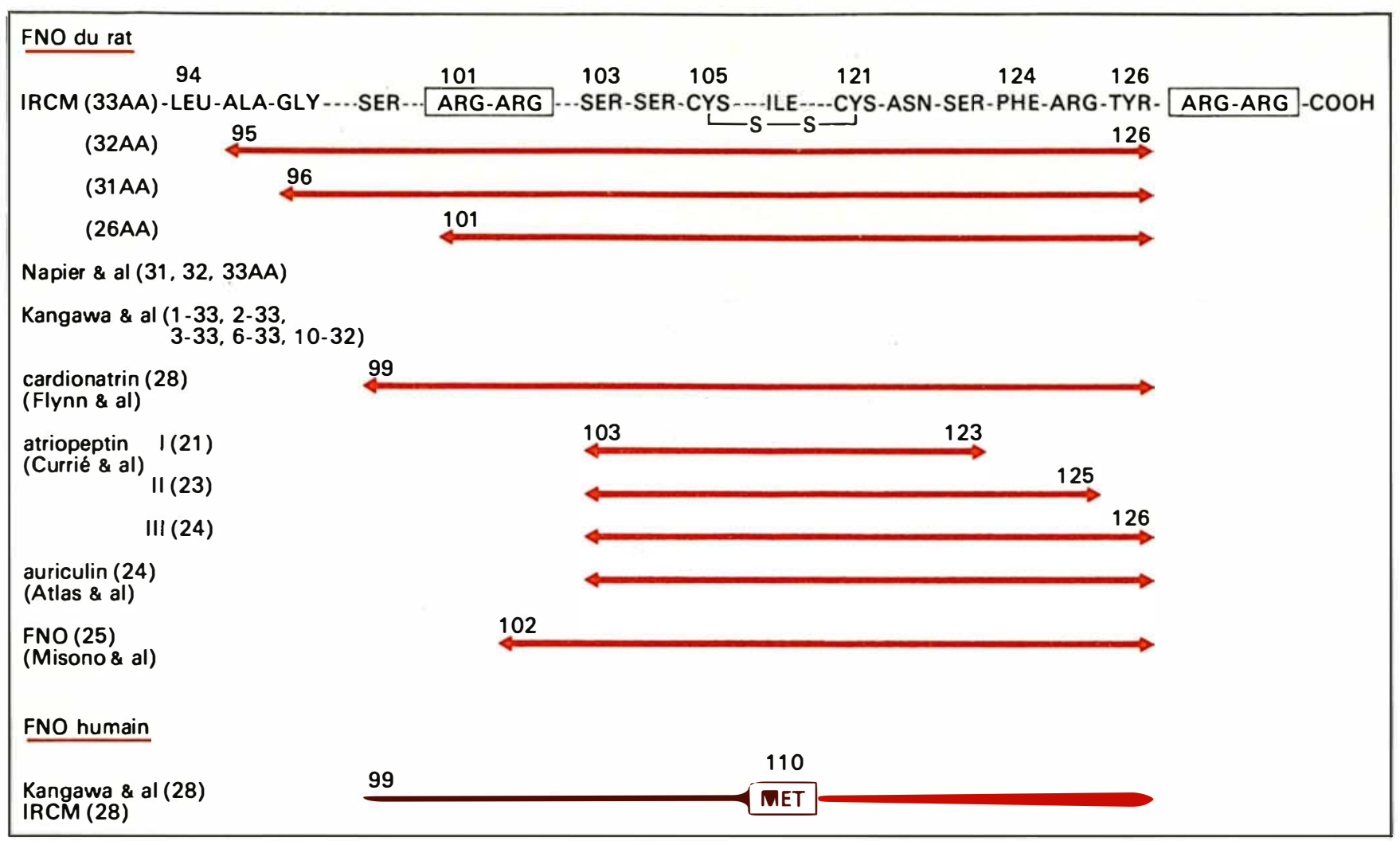

Figure I. Formes actives du FNO de faible poids moléculaire.

des aminés de peptides courts. Nous avons notamment isolé et séquencé un peptide de $33 \mathrm{AA}$ qui fait partie d'une molécule plus longue contenant au moins 106 acides aminés [1] (figure I). Immédiatement après avoir identifié dans nos laboratoires la composition de sa chaîne moléculaire, le peptide 8-33 AA a été synthétisé [15]. Tous les travaux que nous allons maintenant décrire ont été effectués avec ce peptide. I. FNO humain. $\alpha$-FNO humain ( $\alpha$ HANP) a été isolé et séquencé par Kangawa et Matsuo et par notre groupe. Sa structure est identique à celle de l'extrémité C-terminale du peptide de rat à l'exception d'une méthionine qui occupe la même place qu'une isoleucine en position 110.

2. Clonage de l'ADN complémentaire et du gène du FNO. La connaissance de la séquence du peptide de l'oreillette a immédiatement suggéré le clonage de l'ADN complémentaire et du gène du FNO chez l'homme et le rat. L'ADN complémentaire de rat a maintenant été cloné. L'isolement et la structure complète du gène de rat ont aussi été effectués. L'ADN complémentaire pour le FNO humain et le gène humain ont également été décrits. Toutes ces études ont établi que le peptide de rat est composé de 152 acides aminés avec un peptide signal de 24 acides aminés (figure 2 , voir page suivante). Le peptide humain contient 151 acides aminés avec un peptide signal de 25 acides aminés. Le peptide humain ne contient pas les deux Arg-Arg terminaux caractéristiques du C-terminal de rat. Les séquences codant pour le fragment C-terminal et le propeptide précurseur sont hautement conservées chez le rat, la souris et l'homme suggérant que ces structures sont importantes pour leur fonction. Le pont disulfure entre les deux cystéines du C-terminal est essentiel pour son activité biologique. Des expériences effectuées avec le peptide naturel ont montré que des longueurs variées sont actives à condition que le fragment C-terminal soit présent. Généralement, pour tous les organes cibles étudiés, l'activité biolog:que du peptide est d'autant plus faible qu'il est plus long.
3. Localisation chromosomique du gène du FNO.La localisation chromosomique du gène codant pour le peptide humain a été faite par hybridation in situ d'une sonde tritiée à une préparation chromosomique humaine et analysée à l'aide du transfert - par la technique de Southern - d'un hybride cellulaire somatique avec un chromosome 1 normal et réarrangé. Le gène humain a aussi été localisé dans le bras court du chromosome 1 au niveau de.la bande $1 P_{36}$. L'analyse par la méthode de transfert de Southern d'hybrides de cellules somatiques (souris $\mathrm{X}$, hamsters chinois) a été employée pour localiser le gène de souris au chromosome 4 [2].

4. Forme circulante du FNO. La forme circulante du FNO a été isolée du plasma de rats traités à la morphine (l'injection de morphine produit une énorme augmentation du FNO circulant), par extraction, à l'aide de billes de verre Vycor, de chromatographie d'affinité et de chromatographie liquide inverse à haute pression. La séquence en phase gazeuse a révélé un peptide 


\begin{tabular}{|c|c|}
\hline $\begin{array}{lr}\text { peptide } & \text { rat } \\
\text { signal } & \text { humain }\end{array}$ & 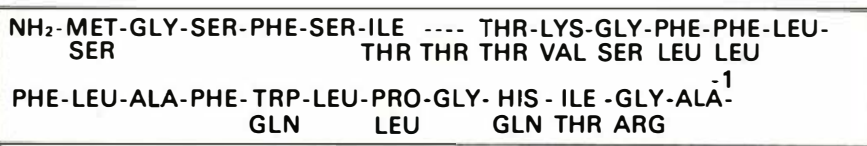 \\
\hline $\begin{array}{l}\text { peptide } \\
\text { de jonction }\end{array}$ & 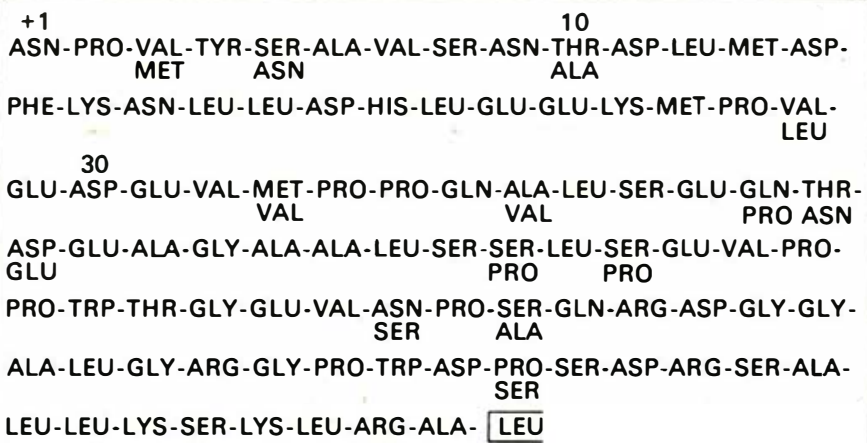 \\
\hline FNO & 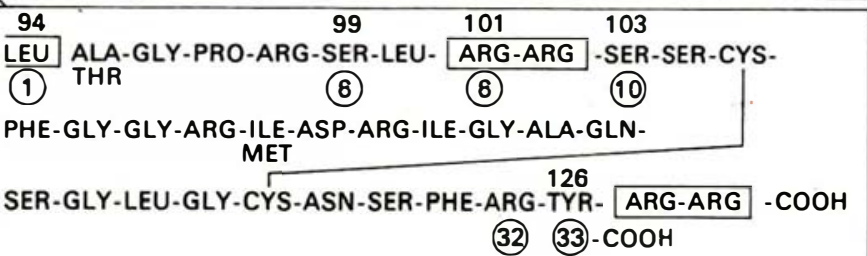 \\
\hline
\end{tabular}

Figure 2. Comparaison du pré-propeptide humain et de rat (d'après Zivin et al., I984; Nemer, Drouin et al., I984).

Sites de protéolyse potentielle. $\bigcirc$ Ces nombres furent employés quand seule la séquence de c-terminus du FNO était connue.

de 28 acides aminés correspondant au FNO Ser 99-Tyr 126 . Son activité est identique à celle du FNO synthétique (Arg 101-Tyr 126) sur la natriurèse, la diurèse, l'effet vasorelaxant, l'inhibition de la sécrétion basale d'aldostérone des cellules de la zona glomérulosa de rat et sur la courbe de déplacement de ${ }^{125} \mathrm{I}$ FNO des récepteurs au FNO des cellules musculaires lisses de l'artère mésentérique. Les surnageants de cultures de cardiocytes de rats jeunes (3-5 jours) ou adultes ont été traités de la même façon pour révéler un peptide de 26 acides aminés correspondant à Arg 101-Tyr 126. 5. Relations structure-activité. Le FNO synthétique (Arg 101-Tyr 126) a été scindé par dégradation de type Edman au niveau du fragment $\mathrm{N}$-terminal ou par des carboxypeptidases au niveau du C-terminal. Les formes plus courtes résultant de ces traitements ont été identifiées par analyse de leurs acides aminés. L'activité biologique de ces formes courtes a été comparée à celle du FNO synthétique (Arg 101-Tyr 126) : tous les tests in vivo (diurèse et natriurèse), ainsi qu'in vitro (activité spasmolytique des artères et du rectum de poussin, inhibition de la sécrétion d'aldostérone de la zona glomerulosa, liaison membranaire) ont révélé que le FNO Arg 101-Tyr 126 est plus puissant que les autres peptides isolés et plus courts, comme par exemple l'auriculine ou les atriopeptines.

\section{Au niveau du rein}

I. Étude des récepteurs. L'injection de ${ }^{125} \mathrm{I}-\mathrm{FNO}$ (Arg 101-Tyr 126) dans l'aorte du rat a révélé la présence de nombreux sites récepteurs dans toute la vasculature corticale du rein mais particulièrement dans les glomérules où les anses capillaires étaient fortement marquées. Des récepteurs ont aussi été mis en évidence sur les cellules endothéliales de plusieurs vasa recta de la médullaire et de la papille. Une étude ultrastructurale comparative de la liaison de ${ }^{125} \mathrm{I}-\mathrm{FNO}$ et de 125I AII (angiotensine II) aux glomérules de rat a révélé que la majorité $(63 \%)$ du FNO se lie aux cellules épithéliales des capillaires alors que la majorité (60\%) de l'AII se lie aux cellules mésangiales.

La liaison aux récepteurs membranaires in vitro a aussi été étudiée dans différentes cellules rénales du chien; alors qu'aucune liaison n'a été observée dans les membranes des tubes proximaux, on a observé cette liaison surtout dans les membranes du glomérule et dans celles de l'anse large de Henle et des tubes collecteurs.

2. Études métaboliques. Des études métaboliques sur des segments isolés du néphron de chien ont aussi été effectuées (en collaboration avec le groupe de P. Vinay). Le FNO synthétique n'a produit aucun changement dans la consommation d'oxygène ou de substrats (lactate, glutamine) dans les tubes contournés proximaux et les anses de Henle. Dans les mêmes conditions, le furosemide diminue la consommation d'oxygène dans les deux types de tubules. De la même taçon, l'utilisation du glucose ne fut pas changée par le FNO synthétique dans les tubes collecteurs. L'incubation préalable de types variés de tubules avec le FNO et, par la suite, l'exposition de différentes populations à ces milieux n'a produit aucun changement métabolique.

3. Seconds messagers. L'injection de FNO natif ou synthétique (Arg 101-Tyr 126) chez le rat in vivo est suivie d'une augmentation de guanosine monophosphate cyclique (cGMP) dans le plasma et l'urine du rat. Le FNO synthétique augmente aussi les niveaux de cGMP dans des tranches de rein et dans des cultures primaires du cortex rénal. Après incubation avec le FNO synthétique (Arg 101-Tyr 126), aucun effet n'a été observé dans les tubes contournés proximaux, une augmentation du double des niveaux de cGMP a été trouvée dans l'anse large et une élévation du triple dans les tubes collecteurs. Le changement le plus important a été observé dans les glomérules où l'on a trouvé une augmentation de 
50 fois les valeurs de base de cGMP au bout de 1 à 2 minutes seulement après l'addition du FNO. Les résultats obtenus en l'absence ou en présence d'une inhibition de la phosphodiestérase appuient l'hypothèse selon laquelle les effets du FNO passent par une stimulation de la guanylate cyclase, plutôt que par une inhibition de la cGMP phosphodiestérase. La distribution de l'activité de la guanylate cyclase dans les fractions du rein fut alors étudiée. Au niveau des glomérules, plus de $80 \%$ de la guanylate cyclase se présentait sous forme particulaire (et non soluble) alors que seulement $20 \%$ de la guanylate cyclase particulaire était présente dans les fractions des tubes collecteurs et des anses de Henle. Au niveau des tubes contournés proximaux, l'activité de la guanylate cyclase était faible et la fraction particulaire n'était pas détectable. Des études ultérieures ont montré que le FNO synthétique (Arg 101-Tyr 126) active sélectivement la guanylate cyclase particulaire et non sa forme soluble au niveau des glomérules, des anses de Henle et des tubes collecteurs. Tel n'est pas le cas dans les tubes contournés proximaux du rein de chien.

A l'opposé, dans les mêmes préparations de rein de chien, le FNO synthétique (Arg 101-Tyr 126) inhibe l'activité de l'adénylate cyclase de $45 \%$ dans les glomérules et les tubes collecteurs avec un $\mathrm{K} i$ apparent de $10^{-10} \mathrm{M}$ à $10^{-9} \mathrm{M}$ et de $30 \%$ dans les anses de Henle avec un $\mathrm{Ki}$ apparent de $10^{-10}$ à $5 \cdot 10^{-5} \mathrm{M}$ mais non dans les tubes contournés proximaux. Le FNO synthétique inhibe aussi, dans les mêmes structures, l'action stimulante de différentes hormones sur l'adénylate cyclase.

Ces résultats semblent indiquer que les effets rénaux du FNO passent, en grande partie, par des changements hémodynamiques au sein des glomérules et des vaisseaux. Les effets du FNO peuvent s'exercer par l'intermédiaire d'une activation sélective de la guanylate cyclase particulaire et par une inhibition de l'adénylate cyclase. L'augmentation du taux de filtration glomérulaire peut provenir non seulement de changements dans l'état de contrac- tion ou de relaxation des artérioles afférentes et efférentes mais encore d'effets directs sur le coefficient de filtration glomérulaire, $\mathrm{K} f$. Un effet direct sur la cellule du néphron proximal n'a pas été démontré; par contre le FNO pourrait avoir un effet direct au niveau de l'anse de Henle et des tubes collecteurs.

\section{Au niveau des vaisseaux sanguins}

Une liaison spécifique de ${ }^{125} \mathrm{I}-\mathrm{FNO}$ a été trouvée dans la vasculature (artère mésentérique et rénale de rat). La déplétion sodée ne produit aucun changement dans les sites de liaison du lit vasculaire mésentérique. L'hypertension rénale (un rein ou deux reins) réduit la densité des sites de liaison, ainsi que la sensibilité vasculaire au FNO. Le FNO synthétique (Arg 101-Tyr 126) diminue l'activité de l'adénylate cyclase dans les vaisseaux sanguins; il inhibe les effets stimulants d'hormones (isoprotérénol, adrénaline, noradrénaline, dopamine) et d'agents comme le fluorure et la forskoline qui activent l'adénylate cyclase. L'effet du FNO synthétique s'additionne à l'action inhibitrice de l'AII sur l'adénylate cyclase des vaisseaux (aorte de rat) mais augmente le niveau de cGMP par stimulation de la guanylate cyclase particulaire.

En fait, le FNO exerce une action inhibitrice assez générale de l'adénylate cyclase, notamment dans le cortex surrénalien ou la glande pituitaire. Par ailleurs, le FNO active la sécrétion de guanylate cyclase au sein de ces mêmes structures endocriniennes.

\section{Localisation du FNO et de ses récepteurs}

Le IR-FNO a été localisé dans le cœur dans un grand nombre d'espèces par immunohistochimie avec la technique de l'anticorps non marqué de Sternberger. Le IRFNO a été localisé exclusivement dans les oreillettes de mammiferes (incluant celles de l'homme) et dans les oreillettes et les ventricules de toute une variété de vertébrés non mammifères. Au niveau ultrastructural, avec la technique de la pro- téine A-or, le IR-FNO a été localisé dans tous les types de grains de sécrétion (A, B et $D)$ dans les cardiocytes des deux oreillettes.

I. Cerveau. La liaison du ${ }^{125} \mathrm{I}$ FNO (Arg 101-Tyr 126) a été évaluée par la technique d'autoradiographie in vitro dans des cerveaux de rats et de cobayes. La distribution autoradiographique des sites de liaison du ${ }^{125}$ I-FNO dans le cerveau de ces animaux est unique et tout à fait distincte de celle des sites récepteurs des autres peptides. Des sites de liaison à haute densité ont été localisés dans l'organe sousfornical, l'éminence médiane, l'area postrema et le noyau du tractus solitaire. Des sites de plus faible affinité ont été localisés dans le tractus olfactif latéral, le noyau sousthalamique et le locus coeruleus. D'autres régions de haute affinité comprennent le bulbe olfactif, le plexus choroïde, le corps ciliaire et l'épendyme. Ces résultats ont été complétés par des études autoradiographiques in vivo chez le rat après injection intracarotidienne de ${ }^{125} \mathrm{I}$ FNO. Ces travaux ont confirmé que les organes circonventriculaires (glande pinéale, area prostrema, organe sous-fornical et l'organum vasculosum de la lamina terminalis mais non l'organe sous-commissural) sont fortement marqués. L'éminence médiane était aussi marquée. Il en est de même pour tous les vaisseaux cérébraux, du plexus choroïde et de l'arachnoïde [I].

2. Autres tissus. Des études autoradiographiques ont confirmé que des récepteurs sont présents dans la zona glomerulosa du rat. On en retrouve aussi par la même technique dans les cellules de la médullosurrénale, de l'endothélium des quatre chambres cardiaques, du foie, du poumon, de l'intestin grêle, du colon et du corps ciliaire.

\section{Implications physiopathologiques}

I. Hypertension. L'injection intraveineuse d'une dose natriurétique $(1 \mu \mathrm{g})$ de FNO synthétique (Arg 101-Tyr 126) produit une chute de la pression artérielle de 6r $\mathrm{mm} \mathrm{Hg}$ dans l'hypertension rénale à deux reins (réninedépendante) et de $45 \mathrm{~mm} \mathrm{Hg}$ dans 


\section{REFERENCES}

I. Cantin M, Genest J. The The heart and the atrial natriurctic factor. Endocr Rez 1985; 6: 10727.

2. Kish B. F.lectron microscopy of the atrium of the heart: Guinca pig. Exp Med Surg 1956; 14: 99-I I 2

3. Jamicson JD, Palade GF. Specific granules in atrial muscle cells. 7 Cell Biol 1964; 23: I5 5 -62.

4. Bencosme SA, Berger JM. Specific granules in mammalian and non mammalian vertebrate cardiocytes. In: Bajusz. E, Jasmin C, eds. Methods and Achievements in Experimental Pathology. Bälc: Karger, 1971; 5: 173-213.

5. Huct M, Cantin M. Ultrastructural cytochemistry of atrial muscle cells. Characterization of the protein content of specific granules. Lab Invest 1974; 30: 525-32.

6. Yunge $L$ Benchimol $S$, Cantin $M$ Ultrastructural cytochemistry of atrial muscle cells. Radioautographic study of synthesis and migration of protcins. Cell Tissue Res 1980; 207: I-II.

7. Yunge I., Benchimol $S$, Cantin $M$ Ultrastructural cytochcmistry of atrial muscle cells. Radioautographic study of synthesis and migration of glycoprotcins. $7 \mathrm{Mol}$ Cell Cardiol 1979; I I : $375-88$.

8. Cantin M, Veilleux R, Huct M. Electron and fluorescence microscopy of the hasmter atrium after administration of 6-hydroxydopaminc. Experientia 1973; 29: 582 .

9. Cantin M, Michclakis AM, Ong H, Ballak M Beuzeron J, Benchimol S. Relationship of specific granules with renin activity in the myocardium. In: Chazov E, Smirnov V, Dhallas NS, cds. Adrunces in Myocardiology. New York: Plenum Press, 1982; 3: 519-20.

ı. Maric JP, Guillemot H, Hatt PY. Le degré de granulation des cardiocytes auriculaires. Etude planimétrique au cours de différents apports d'cau et de sodium chez le rat. Pathol Biol (Paris) 1976; 24 : 549

I I. DeBold AJ. Heart atria granularity; effects of changes in water electrolyte balance. Proc Soc Exp Biol Med 1979; 161: 508-11.

I 2. Thibault G, Garcia R, Cantin M, Genest J Atrial natriuretic factor. Characterization and partial purification. Hy'pertension I983; 5: 74-80.

13. Sonnenberg H, Veress AT, Borenstein HB, deBold $A]$. A rapid and potent natriuretic response to intravenous injection of atrial myocardial cxtract in rats. Phy'siologist 1980; 23: 3

14. Carcia R, Cantin M, Thibault G, Genest J Relationship of specific granules to the natriuretic and diurctic activity of rat atria. Experientia 1982 38: $1071-3$.

15. Scidah NG, Lazurc C, Chrétien $M$, et al Amino acid sequence of homologous rat atria peptides: natriuretic activity of native and synthetic forms. Proc Natl Acad Sci USA 1984; 81: $2640-4$. l'hypertension rénale à un seul rein (non rénine-dépendante). Chez les témoins en revanche, la même dose produit une chute de la pression artérielle de $29 \mathrm{~mm} \mathrm{Hg}$. Ces effets hypotensifs durent moins d'une heure. Chez des rats conscients en hypertension rénale à deux reins, on a fait des perfusions continues pendant 7 jours de FNO synthétique (Arg 101-Tyr 126) $(1 \mu \mathrm{g} / \mathrm{hr} / \mathrm{rat})$ à l'aide de minipompes osmotiques; la pression artérielle initiale de $183 \mathrm{~mm} \mathrm{Hg}$ a diminué graduellement pour atteindre $116 \mathrm{~mm} \mathrm{Hg}$ pendant les deux derniers jours de l'infusion. La diurèse de pression a disparu et la natriurèse de pression a été atténuée. L'activité de la rénine plasmatique est devenue significativement plus basse $(1,81 \mathrm{ng} / \mathrm{ml} / \mathrm{h})$ que chez des rats hypertendus non traités $(8,56 \mathrm{ng} / \mathrm{ml} / \mathrm{h})$. Une régression partielle de l'hypertrophie cardiaque a aussi été observée [1]. Un traitement de plus longue durée (12 jours) avec des doses identiques de FNO (100 ng/h) a produit des résultats identiques. Les niveaux sanguins et urinaires d'aldostérone ont également été considérablement ahaissés. Les mêmes quantités de FNO administrés à des animaux témoins normotendus n'ont eu aucun effet. Chez les rats spontanément hypertendus (SHR) traités de la même façon, la pression artérielle est revenue à la normale, sans changement significatif de la diurèse et de la natriurèse

Chez les SHR, les taux plasmatiques de IR-FNO sont significativement augmentés par rapport à ceux des WKY ou des rats témoins Sprague-Dawley. A l'âge de I6 semaines, la pression artérielle des SHR est de $195 \mathrm{~mm} \mathrm{Hg}$ alors que chez les WKY elle est de $116 \mathrm{~mm} \mathrm{Hg}$. Cette hausse de pression artérielle est accompagnée d'une augmentation significative de IR-FNO (SHR : 203 pg/ml; WKY $128 \mathrm{pg} / \mathrm{ml}$ ). Il n'y a pas de différence dans les niveaux de IR-FNO dans l'oreillette droite: (SHR : 3,31 $\mu$ g/oreillette; WKY : 3,72 $\mu \mathrm{g} /$ oreillette); on note une diminution significative de IR-FNO dans l'oreillette gauche des SHR (SHR : 1,42 $\mu \mathrm{g} /$ oreillette; WKY: 2,28 $\mu \mathrm{g} /$ oreillette). Cette diminution des niveaux de IR-FNO de l'oreillette gauche peut être reliée à l'augmentation de la pression intra-auriculaire gauche chez ces animaux hypertendus.

Ces résultats suggèrent que l'augmentation de pression dans les oreillettes est responsable de l'augmentation des niveaux plasmatiques de IR-FNO et que la diminution en IR-FNO dans l'oreillette gauche est un phénomène de déplétion. Ils indiquent aussi que l'hypertension survient dans ces modèles expérimentaux malgré une augmentation des niveaux plasmatiques de FNO.

2. Insuffisance cardiaque. La présence dans une souche consanguine de hamsters de Syrie d'une cardiomyopathie spontanée et héréditaire conduisant à l'insuffisance cardiaque a fourni un modèle unique pour étudier le rôle du FNO dans cette maladie. Chez les animaux de cette souche, l'insuffisance cardiaque évolue lentement et progressivement, par étapes distinctes. La pression artérielle chez ces animaux est toujours plus basse que che $z$ les hamsters témoins; les pressions veineuses centrales et périphériques augmentent parallèlement avec la sévérité de la maladie. En accord avec les études antérieures faites à l'aide d'un dosage biologique, nous avons trouvé qu'à tous les stades d'insuffisance cardiaque, la quantité de IR-FNO dans les oreillettes est plus basse que chez des hamsters témoins du même âge (200-280 jours). Ces résultats ont été confirmés par des études immunohistochimiques (technique de Steinberger) avec des anticorps monoclonaux dirigés contre le FNO synthétique (Arg 101-Tyr 126) qui ont montré une diminution marquée de l'immunoréactivité des cardiocytes atriaux. L'observation la plus frappante fut une augmentation significative des niveaux de IR-FNO dans le plasma des animaux avec cardiomyopathie. Déjà en insuffisance cardiaque légère, le IR-FNO était en moyenne de $183,7 \mathrm{pg} / \mathrm{ml}(38,4 \mathrm{pg} / \mathrm{ml}$ chez les témoins). Le niveau de IR-FNO s'élève à $257,5 \mathrm{pg} / \mathrm{ml}$ en insuffisance cardiaque modérée et descend à $141,5 \mathrm{pg} / \mathrm{ml}$ en insuffisance cardiaque sévère. Des études 
ultrastructurales ont montré une augmentation extraordinaire du complexe de Golgi des cardiocytes atriaux des hamsters cardiomyopathiques à tous les stades d'insuffisance cardiaque mais particulièrement en insuffisance cardiaque sévère. A ce moment, le nombre de granules de sécrétion est moindre chez les cardiomyopathiques que chez les témoins. De telles modifications ne se trouvent pas dans les cardiocytes ventriculaires. Ainsi, au cours de l'insuffisance cardiaque, une augmentation de la pression veineuse centrale est accompagnée (on le présume) d'un "étirement" des cardiocytes atriaux qui répondent à ce stimulus par une hypersécrétion. Celle-ci conduit à une déplétion progressive du FNO dans les oreillettes et à des images "d'hypertrophie et d'épuisement" de l'appareil responsable de la synthèse du peptide.

Ces résultats indiquent aussi que la rétention d'eau et de sodium, aussi bien que l'activation du système rénine-angiotensine-aldostérone

chez les hamsters cardiomyopathiques survient en dépit de taux circulants accrus de FNO.

3. Expansion du volume sanguin. L'expansion du volume sanguin chez le rat Sprague-Dawley $(\mathrm{NaCl} 0,9 \% ; 0,33 \mathrm{ml} / \mathrm{mn}$, par la veine jugulaire pendant 1 heure) produit une augmentation marquée de la diurèse et de la natriurèse qui débute 5 minutes après le début de la perfusion et qui augmente constamment. La diurèse et la natriurèse sont inhibées de 50\% si l'on injecte à l'animal, au début de la perfusion saline, un antisérum préparé chez les lapins contre le FNO synthétique (Arg 101 - Tyr 126). Le sérum préimmun n'a pas cet effet. On ne trouve aucun changement significatif de la pression artérielle chez ces animaux. Les niveaux de FNO plasmatique s'élèvent de façon significative au cours de la perfusion.

\section{Conclusion}

Des recherches entreprises au cours des 5 dernières années ont établi que les oreillettes du cœur des mammiferes constituent une glande endocrine qui synthétise un peptide de composition connue dont l'ADN complémentaire et le gène ont été clonés. Cette glande endocrine secrète dans le plasma un peptide de 28 acides aminés correspondant au fragment C-terminal de la molécule (Ser 99-Tyr 126). Des études récentes ont montré que le IR-FNO est aussi présent dans les glandes salivaires et dans plusieurs structures nerveuses du cerveau. Le FNO agit non seulement sur le rein mais aussi sur le cortex surrénalien, les vaisseaux sanguins et l'hypophyse. Dans le rein, les poumons, le foie, le cortex surrénalien et le cerveau, tout le système vasculaire comme le montre l'autoradiographie, est marquée. Ces résultats suggèrent que le FNO possède d'importants effets hémodynamiques sur le rein, le poumon, le foie, le cortex surrénalien et le cerveau, qu'il peut contrôler le transport de l'eau et des ions au niveau de l'intestion grêle, du corps ciliaire et du plexus choroïde et qu'il peut avoir des effets métaboliques dans le foie. Les effets du FNO au sein du système nerveux central nécessitent des études plus poussées mais les résultats actuels suggèrent qu'il pourrait exercer une action dans le contrôle central de l'homéostasie du volume extracellulaire et de la pression artérielle. Les effets du FNO peuvent s'exercer, du moins en partie, par l'activation de la guanylate cyclase particulaire et par l'inhibition de l'adénylate cyclase. Des études physiopathologiques ont révélé une hypersecrétion de IR-FNO dans l'hypertension artérielle d'origine rénale, dans l'hypertension spontanée du rat, dans l'insuffisance cardiaque expérimentale et au cours de l'expansion $\mathrm{du}$ volume sanguin. L'hypertension expérimentale, d'origine rénale aussi bien que spontanée est abolie par une perfusion chronique du peptide, probablement par un effet direct sur les vaisseaux sanguins. Ces résultats suggèrent que le stimulus majeur de la sécrétion du peptide est l'étirement des cardiocytes atriaux. Ils suggèrent aussi que le FNO cardiaque et cérébral joue dans l'homéostasie cardiovasculaire (contrôle du volume extracellulaire et de la pression artérielle) un rôle intégrateur qui reste encore à préciser

\section{Summary}

Two independent series of biomedical investigations have led to the discovery that the atria are a peptide-secreting endocrine gland. The first is mainly morphological and starts with the finding that mammalian atrial but not ventricular cardiocytes contain "dense bodies". These "dense bodies" later called "specific granules" were found to be different from lysosomes, to be made up of proteins and to incorporate both ${ }^{3} \mathrm{H}$-leucine and ${ }^{3} \mathrm{H}$-fucose in a pattern typical of peptidesecreting endocrine cells. The finding that rat atrial granulation varied with the sodium and water balance led to the crucial observation that atrial extracts have natriuretic and diuretic effects. In less than five years, this new natriuretic hormone has been purified, sequenced and synthetized, and its cDNA and gene have been cloned. The ANF gene has been assigned to the distal short arm of chromosome $I$ in band I $P 36$ while the mouse gene is localized in chromosome 4. The native and synthetic hormones exert identical wide ranging effects (possibly through particulate guanylate cyclase stimulation and adenylate cyclase inhibition) on the kidney, blood vessels, adrenal cortex and pituitary. Physiopathologic implications of the hormone in experimental hypertension, congestive heart failure and expansion of blood volume are already beginning to emerge. On the other hand, the search for natriuretic hormones or factors by studies of negative pressure breathing, atrial distention experiments, head-out water immersion, expansion of blood volume, $\mathrm{Na}^{+} / \mathrm{K}^{+}-\mathrm{ATP}$ ase inhibition and parabiosis experiments in Dahl rats has provided a general framework within which to interpret this new cardiac function. 